\title{
Accurate Programming: Thinking about programs in terms of properties ${ }^{* \dagger}$
}

\author{
Walid Taha Veronica Gaspes \\ Halmstad University, Halmstad, Sweden \\ \{Walid.Taha, Veronica.Gaspes\}@hh.se
}

\author{
Rex Page \\ University of Oklahoma, Norman, OK, USA \\ page.ou.edu
}

\begin{abstract}
Accurate programming is a practical approach to producing high quality programs. It combines ideas from test-automation, test-driven development, agile programming, and other state of the art software development methods. In addition to building on approaches that have proven effective in practice, it emphasizes concepts that help programmers sharpen their understanding of both the problems they are solving and the solutions they come up with. This is achieved by encouraging programmers to think about programs in terms of properties.
\end{abstract}

\section{Introduction}

Technical usage differentiates being accurate from being precise. "In the fields of science, engineering, industry, and statistics, the accuracy of a measurement system is the degree of closeness of measurements of a quantity to its actual (true) value. The precision of a measurement system, also called reproducibility or repeatability, is the degree to which repeated measurements under unchanged conditions show the same results. Although the two words can be synonymous in colloquial use, they are deliberately contrasted in the context of the scientific method." [1]

Accurate programming is the idea that thinking about mathematical properties of programs as we are developing them helps us produce better programs. We use the term program in the broadest sense to include software, hardware, protocols, and algorithms in general. With the growing success of what is known as property-based testing, thinking about mathematical program properties has suddenly become much easier and much more accessible than it ever was before. These notes introduce accurate programming using Scala as the programming language for examples, and using the ScalaCheck library for specifying and randomly checking program properties.

\subsection{Why are program properties such an important concept?}

In recent years the techniques for developing software and hardware systems seems to have picked up pace dramatically. This trend has been seen in both broad-market methods (such as static typing, static analysis, unit-testing, extreme programming, and more broadly, test-driven development) as well as special-purpose, high-end methods (such as model checking, other mathematical correctness methods, and clean-room methods). The upside of this progress is that we seem to have both much better understanding of how to build such systems well. The downside is that there has been an explosion in the number of terms and concepts relating to the construction and analysis of software and hardware systems.

\footnotetext{
${ }^{*}$ This work is funded by the Swedish KK Foundation, the Fulbright program, and the US NSF.

†This tutorial is contained in lecture notes entitled "Accurate Programming” by Veronica Gaspes, Rex Page, and Walid Taha, available under the Creative Commons 3.0 Unported License online at accurate-programming.org 
The main point that these notes aim to get across is that the mathematical notion of a property can play a central role in helping us organize our understanding of many of these techniques as well as how they relate to or differ from each other. That is, the notion helps clarify why certain recently developed techniques such as property-based testing [4, 3] bear special promise for improving the state of the art in building computational systems.

Thinking about program properties, especially with the aid of a property-based testing tool, simultaneously

1. reduces the number of defects in our code,

2. enhances our understanding of our code,

3. provides us with a powerful, practical way to gain real, hands-on experience with writing and understanding specifications of program properties, and

4. actually makes it easier to go all the way to mathematically prove the correctness our programs, if and when an appropriate technology for doing that kind of thing is available.

We all know that we program to change the world in some real way. So, why should we bother with trying to think about programs in a more mathematical, more abstract way? In addition to the fact that it will allow us to write programs of significantly higher quality, it also has the immediate benefit of simplifying a lot of concepts, and helping us make a lot of connections between a wide range of concepts that may have previously seemed disconnected.

As an example, let us try to see if thinking abstractly can give us better intuitions about why programming is hard. In abstract terms, "to change the world" means to transform one point of its state space into another. As we program, we quickly build up complex transformations that go far beyond simple intuitions. To manage this complexity, our most important tool is careful reasoning, or, put more bluntly, our brain. While programming and careful reasoning are naturally very closely related, it is a curious fact that traditional programming languages and models tend to obfuscate this relation. Programming, we are often told, is about writing programs, and careful reasoning is the realm of logic or mathematics. Another example is an idea that we hope these notes will drive home, which is that programs not only have mathematical properties but they are also mathematical properties themselves.

Viewing programming from the perspective of properties provides a unifying framework for understand strong connections between a range of widely important computing concepts, including

- Use-cases and use-case analysis

- Unit testing, test-driven development, and continual testing

- Automatic test-case generation

- Types, static type checking, and type inference

- Mathematically stating and proving correctness

- Contracts and dynamic monitoring

\subsection{Properties as a tool for the serious hacker}

Far too often, people talking about production programming seem to think that hacking is a bad thing. But hacking, in the sense of experimental programming, is not only fun but also an extremely useful activity. To see that, however, we need to spell out what we mean by the word and analyze the activity with some care. 
Here, we use the term to mean the activity of sitting in front of the computer, writing programs, running them, seeing what they do, revising them, and repeating the process. Surely, other people will have other definitions, and we will not even argue that our definition is superior or popular. It's just what we mean by the term here.

Hacking is a way of finding out if we can write a program to achieve a certain functionality, and to accumulate knowledge about what the language can do, what the machine or compiler running the language can do, or what we as programmers may be able to code up. When hacking is done in the manner described above, the rapid cycle of program modification and execution can provide us with a high-frequency stream of new discoveries about the system that we are exploring. This is a big part of what makes hacking fun. We are creating experiments, running them, and very quickly learning a lot about a system that we are interested in.

Of course, as an experimental process, hacking does have some weak spots. The good news is that the property-based view of programming can actually help us fix these and turn hacking into an activity that is not only enjoyable but also productive. Here are some of the things that happen during hacking that we do not tend to think about very much. The first is that older versions of programs tend to get thrown away: we usually edit the same file in place. This means that we lose one way in which our thought and reasoning process could have been documented. But maybe the history of the code as it is being edited is not the best record of our reasoning while hacking. In fact, often it is the tests that we run on these programs (as well as the expected outputs that we usually never document) that are probably the best record of our thinking process. Thus, a more serious problem is that we generally do not keep track of the tests that we run. An interesting side effect of this is that often newer versions of the program that we are editing do not pass tests that older versions ran. If we had somehow documented the old test cases, we would have quickly realized that we made a mistake when we made some change as we were editing the program. This means that we will often have the impression that we have tested the most recent version more extensively than we actually have. Worst of all, it is often the case that while hacking we tend to use far too few test cases to really provide us with any assurance about the functionality that we think our code provides.

As we will see in this tutorial, thinking about program properties will help us spell out explicitly the kinds of tests that we would like to run on our programs, and using a property-based framework will allows us to automatically test these properties using a large number of test cases. This means that we can hack much more effectively, end up with well-documented and well-tested code, and be able to explain the results of our hacking to others much more clearly and convincingly.

\subsection{What you can expect to learn from this tutorial}

At a practical level, this tutorial aims to convince the reader that:

- Testing really helps you debug your programs and get them right faster. But, you may say, writing test cases can simply be tedious and boring. Using properties to capture how you expect your code to behave helps you write the code and helps you get a lot more test cases "for free".

- Properties are a key concept for expressing what a program is or should be doing. They are a fundamental tool in any notion of accurate programming because until we have a clear idea of where we are going, it is hard to imagine how we can get there, not to mention check whether we are there or not. Thinking about program properties makes testing much more intellectually satisfying. 
- Testing is much easier and works much better when we do it starting from small pieces of code and work our way up to the full system. The "bottom-up" or"from-the-ground-up" approach is the most effective approach to accurate programming. It captures what can be viewed as a divideand-conquer strategy to software quality. It's hard to make high-quality systems from poor-quality ingredients. Interestingly, mathematically proving properties of smaller programs is also much easier. So, if your goal is not only extensive testing but also to mathematically prove the correctness of your code, your best strategy is the same: write and check properties of pieces of your code from the ground up.

- Examples and use cases are crucial for effective software development and are your entry point into the world of properties. The process of developed examples and use cases starts both the process of developing your test suite and of capturing your thoughts about your program in terms of properties. Just as with testing and mathematical analysis, you want to have these for every smallest part of your code. Starting your development by writing representative examples and documenting them in an executable form in your code will save you a lot of headaches when you are checking or proving more sophisticated properties.

At the end of this tutorial, you can expect to be able to:

- Understand why programming is actually quite hard. This is not an immediately obvious fact. Worse, the man on the street is inclined to think that programming is not hard, and that it is just something that teenagers do in their spare time. One of the most fundamental benefits from learning to think about programs in terms of their properties is that we begin to learn the vocabulary needed for reasoning about programs and their behaviors, and what makes some programs easier to reason about and others harder.

- Interact effectively with "customers" and others interested in your programs. Properties provide us with a way to think about our interaction with the "customer" for the code that we are developing, both in terms of soliciting specific use cases and in terms of developing a precise understanding of some of the customer's more nuanced expectations about the code that they want.

- Know how to get your programs much closer to being amenable to mathematical proofs. There are several reasons for this. The first is that a program is much easier to prove correct when it does not have bugs. Testing, especially property-based testing, can be very effective at finding bugs. The other reason is that using property-based testing means you also develop the properties along with the code, thus ensuring that you have all the inputs that you need to start the process of mathematically proving that a program is correct.

\subsection{What you will NOT learn in this tutorial}

While the goal of this tutorial is to teach you to think about and express mathematical properties of programs, it is focused on using testing as a practical and lightweight technique for catching obvious cases when these properties do not hold. This process does catch bugs, and does increase the chances that your programs are correct, but it certainly is not a mathematical proof that the properties of your program hold. For several reasons, this tutorial will not explicitly address the issue of proving programs correct. These include the fact that there are already other texts elsewhere about using tools such as the $\mathrm{Z}$ or VDM property languages to prove the correctness of programs. They also include the fact that traditionally the cost of carrying out such proofs has been prohibitively high for most businesses, in terms of both training their personnel to carry out such proofs and the time and effort it takes to compete the 
proofs themselves, as well as to keep them updated when the code changes. At the same time, when tools to automate the proof process exist, they require even more training time. With the property-based testing approaches, we get essentially most of the benefits of formal verification with dramatically less work.

\subsection{Practicalities: Using Scala and ScalaCheck for exercise problems}

This tutorial is written with the intention that the reader will read the notes and use Scala and ScalaCheck to perform the exercise problems presented in the text. Therefore, it is important that you have Scala and ScalaCheck installed and running on your machine before starting to go through the rest of the tutorial.

To use Scala and ScalaCheck [5] you need to download the compiler and some tools that follow with it. The best way is to go to the official site for downloading

$$
\text { http://www.scala-lang.org/downloads }
$$

and follow the instructions for your system. You need to have Java 1.5 or later in your system. If you don't have it, there are instructions on the scala download page. All information and documentation about Scala is under

$$
\text { http://www.scala-lang.org/ }
$$

Together with Scala you get the Scala Bazaar System (sbaz). To start using it, follow the instructions in the first section of

$$
\text { http://www.scala-lang.org/node/93 }
$$

Now, in order to install ScalaCheck, you just have to use sbaz doing

sbaz update

sbaz install scalacheck

All documentation and more detailed instructions are under

$$
\text { http://code.google.com/p/scalacheck/ }
$$

In order to test your installation, you can write a Scala program

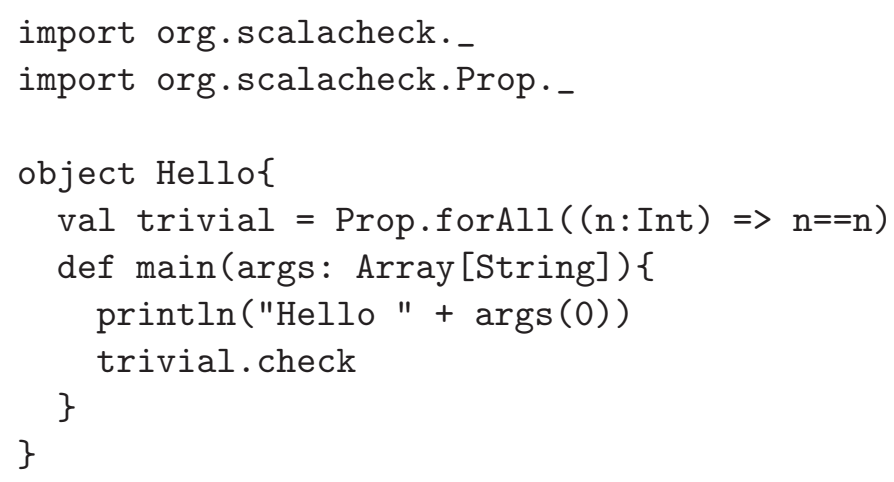


If you have any problems running this program, it could be the case that sbaz did not find the latest release of ScalaCheck. Then you have to do some work by hand! Under the directory

.../scala-2.9.0.final/lib

place the file you find under

http://scalacheck.googlecode.com/files/scalacheck_2.9.0-1.9.jar

with the name scalacheck.jar.

\section{A Simple Function}

Consider the following programming task: write a function that takes two integers and returns the larger of the two. Even though this is a simple problem, it can be used to illustrate a wide range of best practices and concepts relating to software development.

\subsection{Examples as a communication tool}

Mastering accurate programming requires awareness of several facts that are quite simple but at the same time of tremendous importance in practice. One of these facts is the exceptionally important role that concrete examples play in effective communication. A concrete example is one in which all the details have been spelled out, and nothing is left to imagination or intuition. Here, we also use the word communication in the broadest sense to include writing a letter, giving a speech or presentation, discussing an insurance policy, or talking about computer programs.

There are several reasons why concrete examples are important. The first is that as humans we experience the world primarily through a small set of basic senses. Yet we think about the world and communicate about the world in much more general terms than what our primary experiences are made of. Our beliefs about primary experiences are much easier to communicate than more abstract thoughts about them. For example, it is much easier to reach an agreement about what is a "hot tea pot" than what is a "pretty tea pot", because the first relates directly to our senses, while the latter is a much more sophisticated and context-dependent notion. The same kinds of difficulties arise when we try to communicate about programs.

The second is that using concrete examples reduces our reliance on imagination or intuition, both of which can actually be hard to communicate, especially about complex objects such as programs. Programs are generally deceptively more complex than they appear at first. This makes it particularly important to spell out concrete examples that communicate concrete facts about how we expect such programs to behave.

The third and final reason is that it is too often the case that programs are built with too few test cases. Making a habit of starting the process of creating programs by first creating concrete examples is not only a good way to start understanding the problem that we are trying to solve but also to ensure that there will always be a minimal set of test cases that we can easily use in the future to make sure that our program continues to work correctly as we update it, upgrade it, modify it, or attempt to improve it in any other way.

\subsection{Use cases, use-case analysis, and test-driven development}

The reader may be somewhat surprised by the emphasis we put on this idea. However, it is the key idea behind important methods in software engineering, such as Use-case Analysis [6] and Specification by 
Example [2]. The idea of using examples is very simple, but experience repeatedly demonstrates that they are one of the most important ingredients of successful program development. Not only are examples useful for accurately understanding the functionality that we are being asked to program, but they are also extremely helpful in checking that we have attained and maintained the functionality that we think we have attained. This observation is the basis for widely successful methods of test-driven development, including techniques such as unit testing and extreme programming. Test-driven development promotes the idea of starting development with building an appropriate set of tests for the functionality we want to develop. Unit testing pushes this idea a bit further to emphasize that it is most effective when applied at a fine granularity, so that tests are made when implementing the smallest possible unit of functionality. This idea of improving the quality of software from the ground up is very important, whether we simply want to make sure that we have a high-quality test suite for our code or we want to go all the way to prove our programs correct.

\subsection{Examples as properties}

It is easy to put these ideas to work. For the most part, it is just a matter of making it into a habit. We will do this consistently in this tutorial. For the max problem, we may be able to solicit the following concrete use-cases:

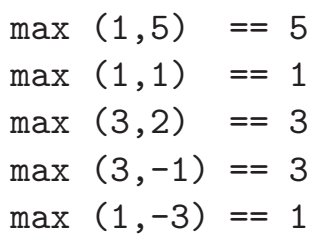

With these concrete examples in hand, we get a more specific idea of what is required. The last example above is actually particularly useful, because it excludes the possibility that the customer actually meant us to return the value with the greatest magnitude rather than simply the greatest value. Naturally, language is very expressive, and much of its expressivity comes from the fact that the meaning of any one sentence can be highly context dependent. Concrete examples can be very helpful in making sure that we are not making any false assumptions about the context.

We can now confidently write out code for an implementation of this function:

def $\max (\mathrm{x}:$ Int, $\mathrm{y}:$ Int $):$ Int $=$ if $(\mathrm{x}>\mathrm{y}) \mathrm{x}$ else $\mathrm{y}$

\subsection{Use cases as properties}

ScalaCheck is a Scala library that allows us to explicitly specify and test a wide range of program properties, including specific test cases. To capture the behavior expressed in the above examples, all we have to write is the following:

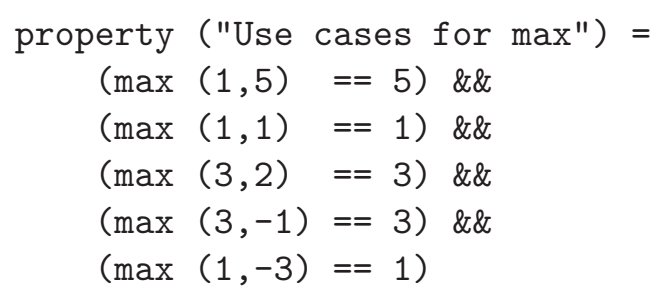


This statement is executable test code. When we run the above code (both definition and property statement), we get the following output when we run our program:

+ SmallExamples.Use cases: OK, proved property.

Not only is this a comforting confirmation that our code at least works on the examples that were discussed with our customer, it means we have an executable test that we can keep in our test harness so that we can always easily detect if any future changes to the code break functionality that we had already gotten right. The idea of keeping such test cases around and treating them as an integral part of the code that we developed is an essential part of test-driven approaches to software design.

At this point we can stop, declare victory, and hand back our code to the customer and say that we are done. While this could be a reasonable thing to do for a small function such as this one, for many problems we may want to study our own code a bit further to make sure that we really understand what it is doing. Reading the code and giving it to other people to read is always useful. But this short example also suggests that we and the other readers may feel that we don't necessarily know everything about the primitive operations used in this one-line program, so it is useful to produce more test cases to test some general properties that we expect to hold for this function. An example of such a property could be that we expect that this function is symmetric-that is, it produces the same answer even if we switch the arguments around. We can use concrete numbers to test this idea, but it would be even better if we can just write that down in a more general way.

\subsection{Universal quantification}

Mathematics provides a great way to make but still very precise statements like the ones we are looking for. For example, we can express the symmetry property that we require here as saying that we want that for all $\mathrm{x}$ and $\mathrm{y}$ that are Int values it will be the case that $\max (\mathrm{x}, \mathrm{y})$ produces the same number as $\max (\mathrm{y}, \mathrm{x})$. Using more concise mathematical notation, we can write this statement as follows:

$$
\forall x, y \in \text { Int. } \max (x, y)=\max (y, x)
$$

Technically, in a statement like the above we are leaving it implicit where the definition of Int and max are being looked up from, but the formulation above captures the gist of what we are trying to get at.

\subsection{Random testing of universally quantified properties}

A universally quantified property such as the one above can be expressed as follows:

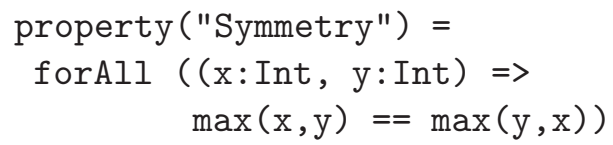

The above statement is just a Scala program which makes some reference to primitives defined in the ScalaCheck library. When we run this program, the system comes back and gives us a more interesting response than the first one:

+ SmallExamples.Symmetry: OK, passed 100 tests.

Here, ScalaCheck generated 100 different pairs of values for $\mathrm{x}$ and $\mathrm{y}$ and used them to test the validity of our property. ScalaCheck gets the hint that we will need it to generate some test integers for us when 
it sees the "forAll" operator. The convenience of the forAll operator comes from the fact that it can alleviate the need for us to actually come up with 100 different pairs of numbers to write down such a test, not to mention writing them down explicitly. Of course, there are many occasions when it is useful for us to write down individual test cases, but there are also many occasions when it is useful to have them be generated automatically. In this case, it is also useful for us to document the symmetry property in our code, and in a manner that can be easily executed whenever we want to check the correctness of our code (which is virtually any time we make any change to it).

We can continue to study our one-line program by considering other properties that it may have. In this respect, it can be useful to think about how such a function could be used. For example, we may use this two-argument max function to keep track of the largest number that we have seen as we go down a list. From this point of view, if the next number we look at is the same as the maximum number we have seen so far, we'd like to keep the maximum the same. The following property tells us that the max function can do this for us automatically:

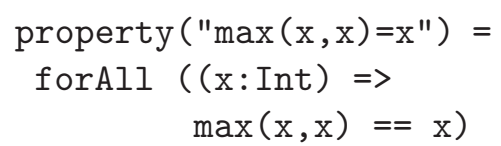

This last property illustrates how understanding the properties of the code that we have written well can often help us fully appreciate the behavior it provides and take full advantage of its functionality to simplify the way we use it.

Another important property of this function is that the result it returns is an upper bound for the two values that it takes as argument. This property can be expressed as follows:

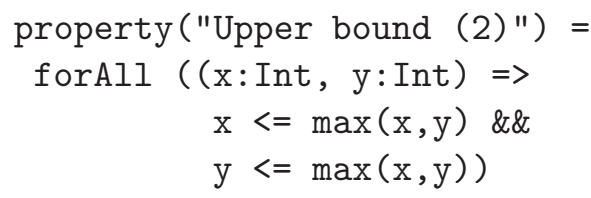

Note that if we take into account the first property that we stated about max, symmetry, then the property above can actually be simplified to consider only one argument. That is, when we have symmetry the property above is implied by the following, more concisely stated property:

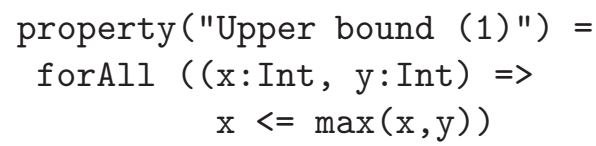

\subsection{Properties that completely characterize a function}

Often, it is useful to consider whether we have been able to express the simplest property that fully characterizes the behavior of the code that we wrote. Interestingly, the above property (and, in fact, even all the properties that we have written above combined) do not fully characterize our max function. In particular, the last property says that the result of max is an upper bound, and not necessarily a particular upper bound. In fact, max computes the least upper bound of two numbers, in the sense that max produces the least number satisfying the above property. This additional constraint can be expressed as follows:

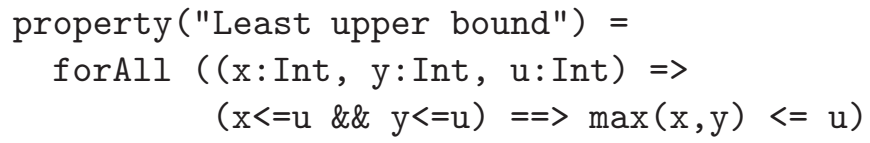


Together, these two seemingly very generic properties actually fully characterize the intended behavior of the maximum function.

Exercise 2.1 Consider the following function:

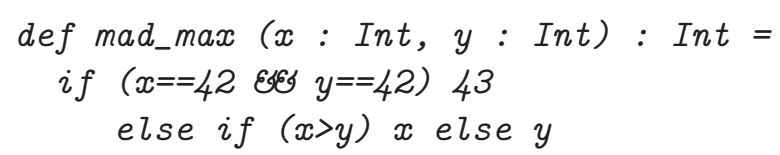

Which of the properties considered above would break if we used this function in place of max? First, write down your answers when you check the properties by hand. Then, run all the tests using this function, and note any differences between what you expected and what you got from running the tests.

\section{Programs as a Special Type of Properties}

It is instructive to note that certain properties can be equivalent, in the sense that they capture the same concept or behavior. It is also useful to note that programs themselves can be viewed as properties. To see this in the case of the max example, we can consider the following property:

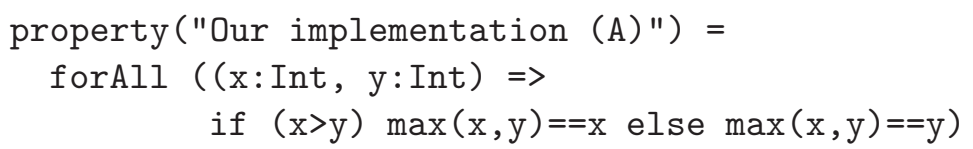

It looks remarkably similar to the way we have implemented the max function. The body of the if statement is not quite the same, but this is only a cosmetic difference. We can express the same property as follows:

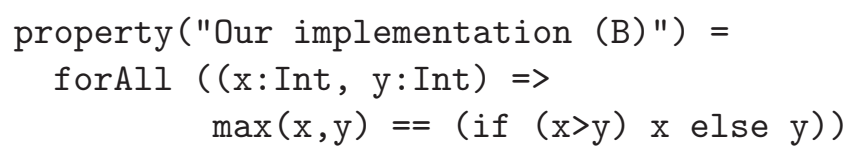

This property essentially gives us the code for our implementation. This is a very useful connection between properties and programs, and there are numerous situations in which converting one property into another equivalent property that has a different form can be a very useful method for deriving programs that implement the properties that we are interested in. Note, however, that this generally requires that we have spelled out a set of properties that we want our program to have. In many instances, however, we need to both develop the code and spell out its properties at the same time. It is also interesting to note that this means that code/property code design is, in a sense, just property design. The useful distinction in that case is that we are generally cross-testing two things against each other, and even though they are both properties, we are generally cross-testing different properties against each other.

\subsection{Properties as relations}

It is reasonable to consider whether there are certain characteristics that make some properties closer to being programs than others. One way to draw this distinction is to recognize that properties are generally mathematical relations between different sets. For example, in the case of max, we wrote many properties that relate two or three sets of integers at the same time. Intuitively, we can view properties as executable when they provide relations that are actually functions (which is a special kind of relation) from elements of a set that we consider to be the input to the computation into finite elements of another set that we consider to be the output of the computation. 


\subsection{Programs as functions}

Often an implementation is the best specification that we can express for a function. This observation has a very real and concrete practical implication, which is that for some programs it is hard to write a specification without spelling out the code of the program itself. This is a mixed blessing. On the one hand, it means that we (either set out to or discover after the fact that what we just did is to) first write the code, and then we start thinking about other, "extra-computational" properties about it that we may want it to have. This can seem strange at first, but it is something that can arise naturally in many situations, and that often produces very useful programs to have around. All it means is that the program that you are describing has computational content that can be elegantly described, and you managed to find it.

On the other hand, this deep observation should be approached with care. In particular, it does not mean that all programs are elegant descriptions of their functionality. Quite the contrary. Given that most programs in the world are buggy, it is highly unlikely that they are an elegant description of anything, or that there are any other properties that characterize their behavior and that could be viewed as elegant. It only means that particular programs also happen to be great specifications of their own behavior. Examples of such programs include definitional interpreters for programming languages and insertion sort as a prototype for sorting.

\subsection{Functions as "The Reference Implementations"}

Usually, such self-evident programs are not simultaneously the best implementations in terms of performance. Often, one still has to do a substantial amount of work to go from an implementation that is a nice specification to an implementation that is efficient. For example, if we are talking about languages, compilers are generally more efficient implementations than interpreters. If we are talking about sorting, there are numerous implementations of sorting that are much more efficient than the elegantly described insertion sort. Yet, in all of these cases, the self-evident or reference implementations are an invaluable tool for developing and testing the correctness of more efficient implementation. For novel algorithms, however, the challenge lies in producing the first such reference implementation in the first place. Thus, emphasis on code vs. property development while developing an algorithm that was not previously specified varies dramatically from the emphasis when we already have a reference implementation and we are trying to build a more efficient one.

It is also useful to note that having the programming language and the property language be syntactically close is also a mixed blessing. On one hand, it can facilitate turning an expression from being a program into a property and vice versa. On the other hand, the intuitive meaning of the expression can also change in subtle ways when we do that, depending on the context.

\section{Functions on Numbers}

The max functions illustrate that even a one-line program can have interesting properties and deserves a reasonable degree of analysis. Maybe more interesting than the fact that it is a one- line program is the fact that it only involved arithmetic (comparisons) and a conditional statement. In practice, many interesting programs involve iteration or recursion, and as a result both perform more computation per line of code and also exhibit more interest properties. 


\subsection{An iterative program}

As a simple example of a problem that requires iteration, consider the following problem: Write down a function that takes one argument, call it $\mathrm{n}$, and computes the sum of the numbers counting up from 0 to $n$.

Before we start writing out examples, it is useful to note that there is implicit information in this problem statement that is useful to spell out. Because the problem says "count up from 0 to n", it is reasonable to assume that our customer is assuming that the number $\mathrm{n}$ is either more than or (at least) not less than 0 . This also means that we are left with the task of determining what the function should do if the input is less than 0 . Now we can start creating some examples to convince ourselves that we understand the function required. We will write it as a property so that it it is easy for us to just run the test when we have written the code.

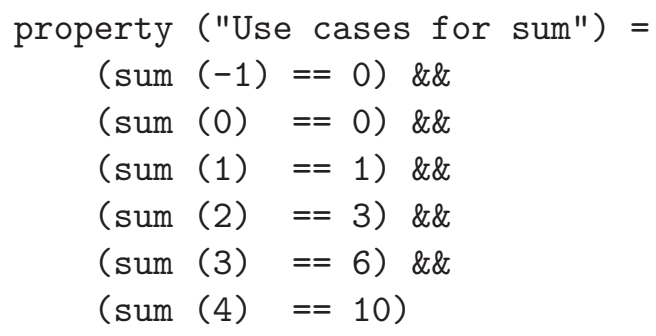

If we are in a hurry, we can decide that we have satisfied the requirements of due diligence, having written out some examples, and then just write down an implementation of this simple function as follows:

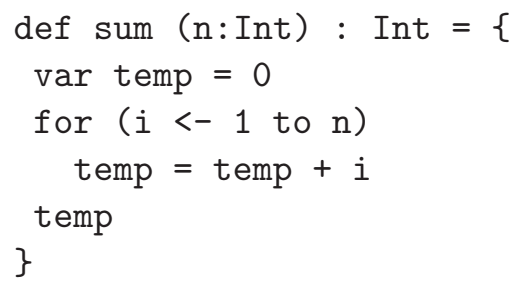

\subsection{Extracting general properties from use cases}

If we have a little more time, we can productively think a bit more carefully about this problem and about the code. In fact, just by listing these examples, an interesting pattern emerges. It's actually easy to compute the next value in this list of examples when we start from 0 (or below) because the result is 0 until we reach 1, and from that point on the result is the argument added to the result of the previous one. For many problems in which we take a positive integer as input, this is an extremely helpful pattern to recognize when we want to write out code that solves a certain problem. The two parts of what we have observed here can be expressed as the following two properties:

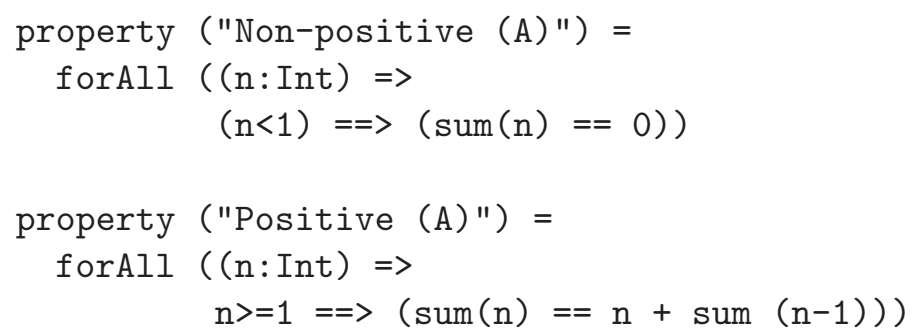


These two properties are quite interesting because they fully characterize the sum function. Not only that, but they can also be naturally rewritten in a step-by-step manner to guide us to executable code that captures the intuitions that we drew from the examples. Granted, this is an extremely simple function that we are being asked to write, but it is useful to reflect on the process of writing, as it were, in "slow motion". And the point is not that this is precisely how all problems should be approached, but rather that the connections between all of these different views of the problems exist, and that these different views are in fact themselves properties with strong connections among them.

\subsection{Reasoning about properties}

The two properties above can be rewritten to look a bit more like code and less like a mathematical property. In particular, we can rewrite them into :

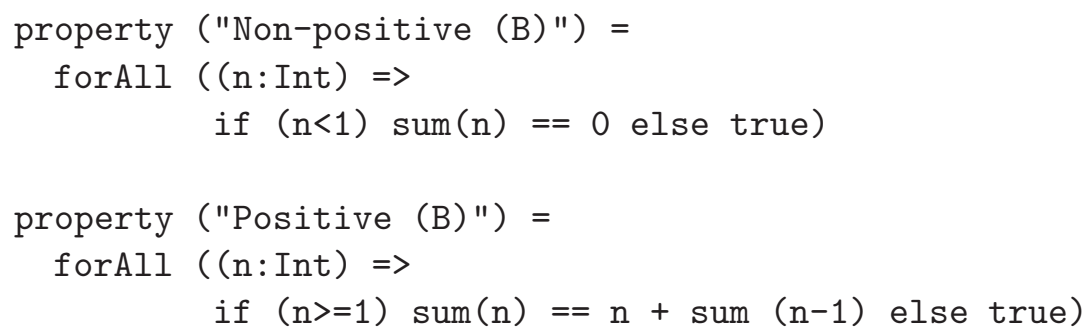

All we did here is replace any implication of the form $A==>B$ into an (if ( $A$ ) B else true). Having true in the else branch of the if is consistent with the way mathematical implication works. When the thing before the implication is not true, we can have anything after the implication and the whole property is still true.

Next, we note that these conditions in the two if statements in each property are complementary. In fact, we can rewrite the first property so that there is even more alignment between the two properties, and so that they can be combined into one:

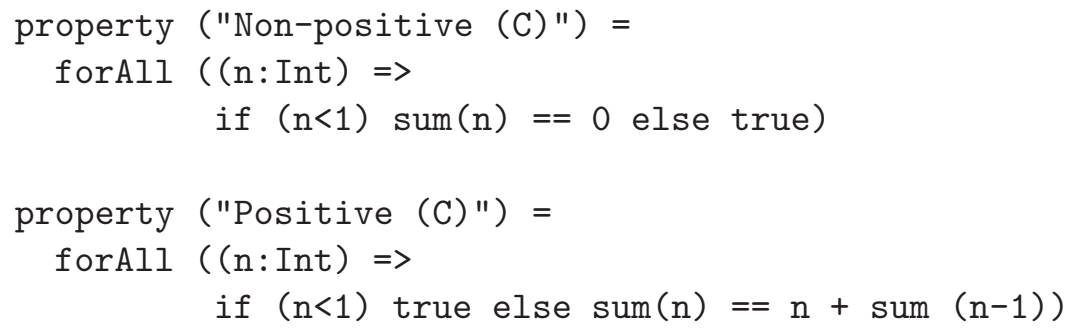

Only the second property was changed, and all that was done is to replace the condition ( $\mathrm{n}>=1$ ) with the dual condition $(n<1)$ and then flip the else and then branches. Note that it is easy to see that both properties have the form of an if statement with exactly the same condition. Furthermore, each one has alternatively "true" in one branch and a more interesting condition in the other branch. In fact, true is the least interesting condition to have in any property, because it is a trivial condition that makes no statements about any variables by itself. Thus, what we can do is to combine both properties into one, keeping only the interesting branch of each property in the result.

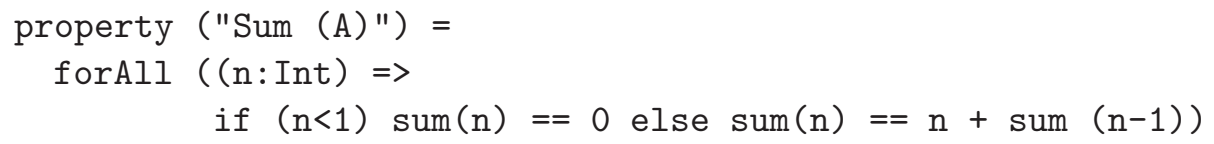


At this point, we are very close to having the exact code for an executable program for computing the sum. All we need is to note that both branches start with the expression $\operatorname{sum}(\mathrm{n})==$, and to reformulate our property one more time to take this equality outside the if statement:

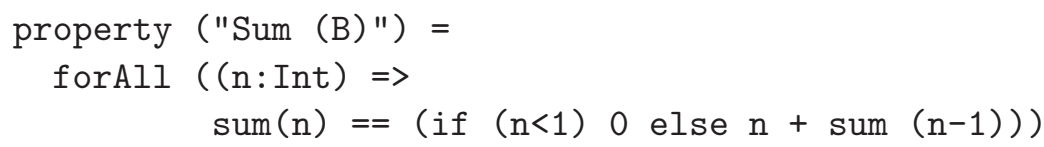

Not only does this leave us with a shorter description of the same property, but we also have an equality test where on one side we have just the term sum(n), and on the other side we have an expression that can be viewed as a perfectly valid inductive definition of sum(n). In particular, the if statement simply returns 0 for all non-positive values, and for positive values, it can compute a result by adding $n$ to $\operatorname{sum}(\mathrm{n}-1)$.

Our original sum function, in fact, passes all the properties that we have seen so far. These tests actually take a substantial amount of time to run, but they all pass. We could indeed declare victory at this point and either go back to the customer and hand over our code or start thinking about other things, like optimizing our implementation.

Exercise 4.1 The following definition for sum can be viewed as more efficient than the one above:

def sum2 $(n:$ Int $):$ Int $=$ if $(n<1)$ o else $(n+1) * n / 2$

Use ScalaCheck to check whether or not it satisfied all of the properties discussed above.

But we can also continue to study our problem and its solution by considering more properties that we expect to hold about the function.

\subsection{Why working with numbers requires special care}

Often, continuing to think carefully about the properties that should hold for a given program requires stepping back to identify what should be the most obvious properties that it should enjoy. One such property is monotonicity-that is, the result of the function should grow (or at least not decrease) as the argument is increased. This property can be expressed as follows.

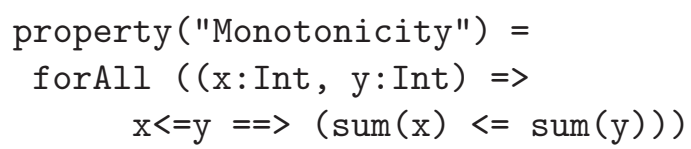

This property actually fails for our implementation. In particular, we get the following output from ScalaCheck:

! SmallExamples.Monotonicity: Falsified after 0 passed tests.

> ARG_0: 0 (orig arg: -2147483648)

$>$ ARG_1: 65536 (orig arg: 660619302)

ScalaCheck is saying that the property fails, and in particular that values 0 and 65536 for $\mathrm{x}$ and $\mathrm{y}$ demonstrate that this property fails. We can get a better idea of what this test has uncovered by inspecting the results that our sum function returns for these values:
println (sum(0))
$/ /$ prints 0
println (sum(65536))
$/ /$ prints -2147450880 
By stating our monotonicity property, we have uncovered an overflow problem. The test points us to the fact that the Int type is finite and uses a fixed number of bits to represent just a subset of the integers. When we add one too many numbers, the result is a value that is not representable, and we get instead another, meaningless value.

It is highly instructive to note that we had already expressed several properties up to this point, and they all passed the test and were all valid. But they were not really enough to help us realize that our implementation had limitations that we may have not thought of before. This example also illustrates that the properties we express may not always be saying precisely what we thought they were saying when we wrote them. In this instance, part of the problem comes from the fact that we used the same language for both the program and the properties, and both including reference to the finite type Int and the same definitions for the arithmetic and comparison operators defined on that type.

Exercise 4.2 A smart programming language that supports recursion would allow us to use a definition like ("Sum (B)") directly as code, and so we can define our function simply by copying the above if statement into its body as follows:

$$
\text { def sum3 }(n: \text { Int }): \text { Int }=\text { if }(n<1) \text { o else } n+\operatorname{sum3}(n-1)
$$

This is a reasonable solution for the problem that we set out to solve. However, while the reasoning with which we arrived at this solution is perfectly valid under the right assumptions, the fact that we have expressed the various intermediate properties that lead to this solution in an executable form allows us to use ScalaCheck to reveal to us that our assumptions were not completely valid.

Use ScalaCheck to test that the above recursive definition for sum actually satisfies all of the properties that we described. If any of the properties break, explain why, and show how to solve this problem.

\section{Functions on Aggregate Types (I)}

The way we develop and think about programs is affected by the type of data structures on which they operate. So, when we work with Int values, many of our properties will look a lot like the kinds of properties that we see in traditional math courses, such as symmetry, monotonicity, and so on. When we work with other data structures, such as lists for example, there will still be interesting patterns to the mathematical properties that we use to describe the behavior of such programs, but these patterns may seem different from the ones that we have seen in high school math classes. As we see more examples of using properties to describe and reason about programs, clear patterns will definitely emerge.

\subsection{Surface syntax for lists}

Aggregates or collections are data structures that bring together several small units of data. A basic data structure is the list. In Scala, we construct lists by writing something like List $(1,2,3)$ to represent a list of three elements, all integers, and in this case consisting of the elements 1,2, and 3.

\subsection{Deeper structure and inductive nature of lists}

Aggregate types, including lists, are often inductively defined. Knowing that a type is inductively defined is very helpful both for writing programs that take values of this type as input and for thinking about the kinds of properties that such programs should have. Write List $(1,2,3)$ is in fact syntactic sugar for the more primitive list constructors, which would express the same list as $1::(2::(3:: \mathrm{Nil}))$, where Nil is the 
empty list, and the operator (::) is the constructor for non-empty lists, which takes an element and a list as an argument. Knowing the names of these primitive constructors for lists allows us to use the Scala match statement to write a function that checks whether a list is empty or not:

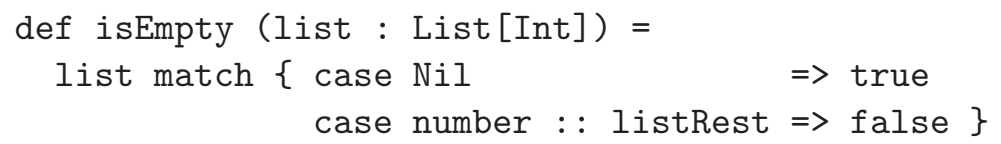

The match statement not only allows us to check which constructor was last used to build the list but also allows us to refer to the components of the non-empty constructor (by the names "number" and "listRest" in the second branch of the case if we want to).

\subsection{Use cases for a function on lists}

With these primitives in hand, we can approach our first programming task relating to lists: develop a function "count" that takes an integer and a list of integers and returns a count of the number of times this integer occurs in the list.

To check our understanding of the required functionality, we write down several examples. In practice, writing down examples is a useful thing to do while still with the customer discussing requirements, and also at the start of programming to make sure that we have a clear understanding of the task at hand.

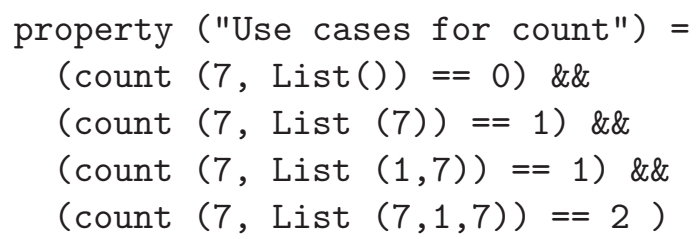

\subsection{Defining a function on lists}

To think clearly about a new function that we have been asked to write, it is actually very useful to keep in mind the properties of the type of value it takes as input. If the input type is inductively defined, which is the case for lists, this generally means that we should use a match statement for case analysis and recursion that (often directly) mimics the pattern of recursion in the inductive definition of the type itself. In the case of this task, we can implement the function count as follows:

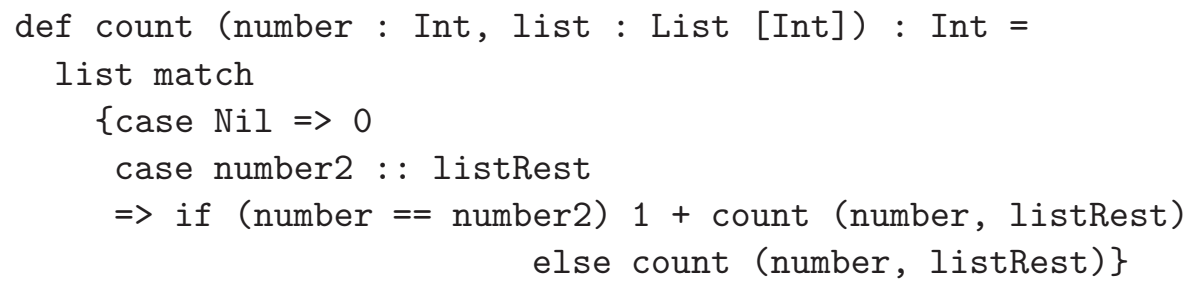

The code above passes our test examples, so we can turn to thinking about some deeper properties that can give us further assurance about our implementation.

\subsection{A non-defining property}

One property of this function is that count for a number in two appended lists should be the same as the sum of the count in each of the two lists individually. The notation for appending lists is simply (++), so we can express this property as follows: 


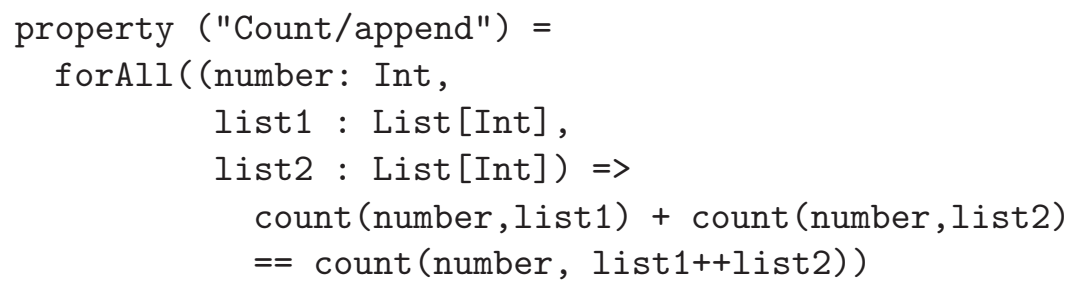

While the above property is highly generic, it can be satisfied by an implementation that is certainly not correct. In particular, if we used a faulty implementation that always returns zero, it would also satisfy the property above. To avoid this kind of problem, it is useful to add a property that captures our intuition that adding another instance of the number we are looking for into the list increases the count by one. The following is an example of a property that captures this fact:

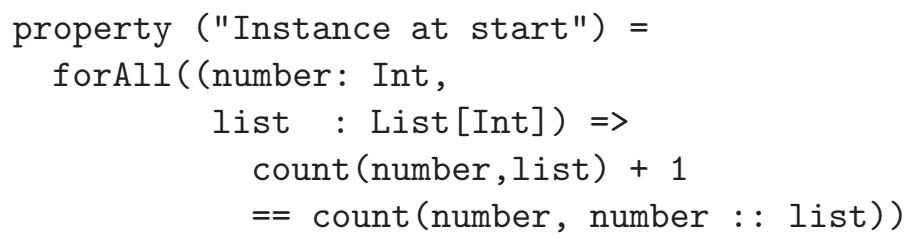

Adding this property to our specification of the functionality of the counting function gives us much more information about our implementation. But it is useful to realize that it is not a complete specification of counting.

Exercise 5.1 Check that the following function satisfies both the "Count/append" and the "Instance at start" properties:

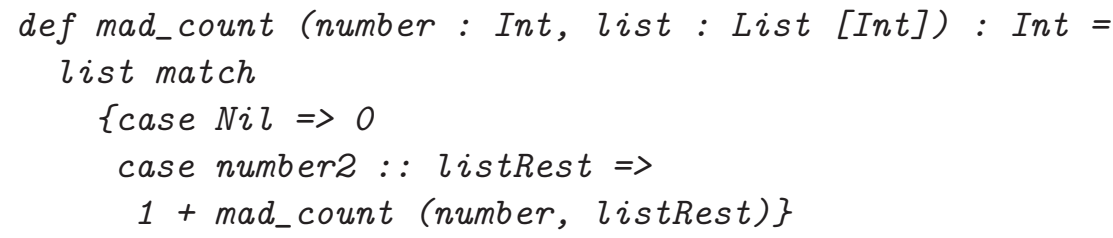

If the function above satisfies those two properties, suggest at least one new function that also satisfies these two properties.

One thing that the "Instance at start" property does not capture is that if the instance added to the start of the list is not the same number, then the total count should not change.

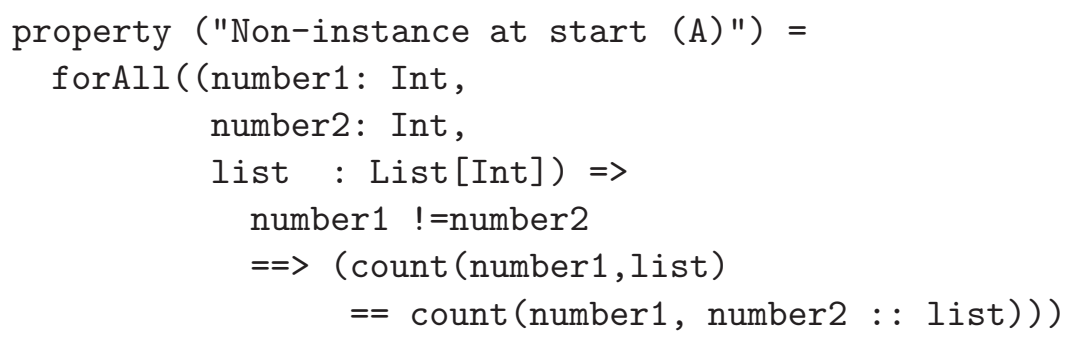

If we rewrite this property to replace the implication with an if statement, it will be easier to see that this and the "Instance at start" property can in fact be combined: 


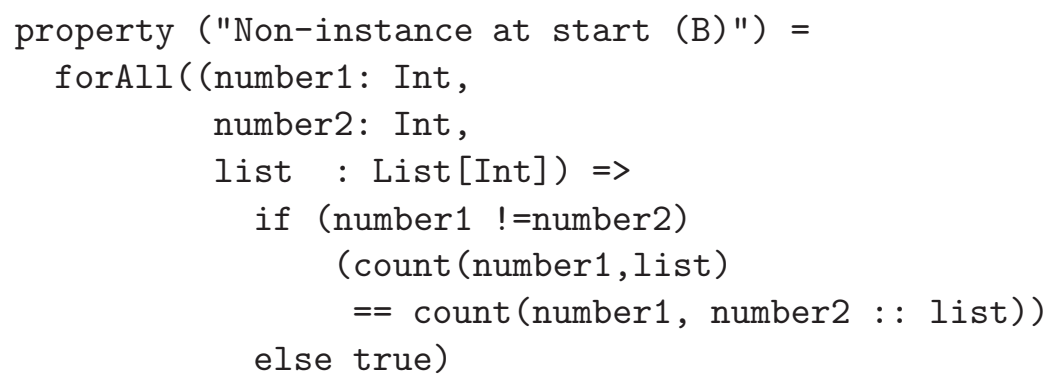

And now we can replace the "true" branch with the property that we expect to hold when the two numbers are equal, and we have:

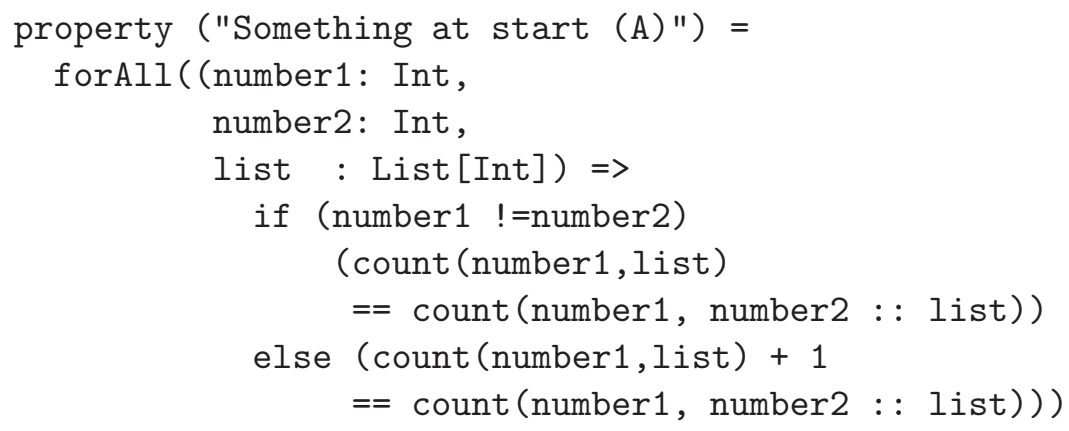

We note well that we have " +1 " in the statement in the second branch of the if statement.

Clearly, this property combines the power of our two past properties about what happens when we add an element to the list. However, it still does not really fully specify the behavior of the counting functionality. It may be surprising to the reader, but it still leaves an unbounded amount of freedom in the behavior of the function "count". In particular, the following function would actually satisfy all of the properties that we have expressed so far:

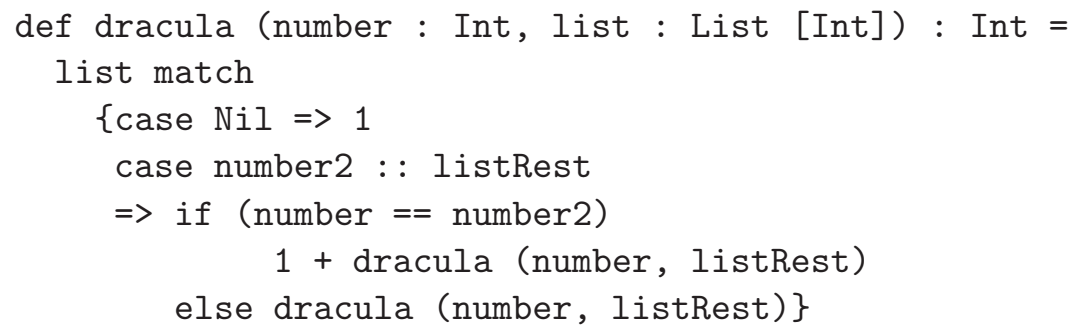

And, in fact, any version of the above function with any integer value in the case of the empty list (Nil) would have satisfied all of the properties that we have expressed so far. We can incorporate this final, additional requirement into the last property by introducing an additional check for the case in which (list $=$ Nil) and rewriting it as follows:

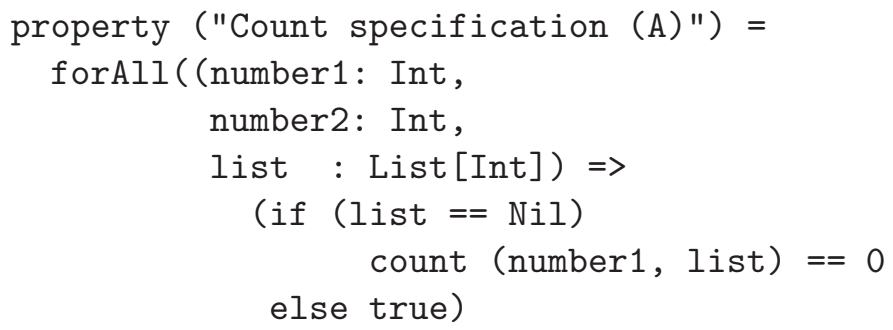




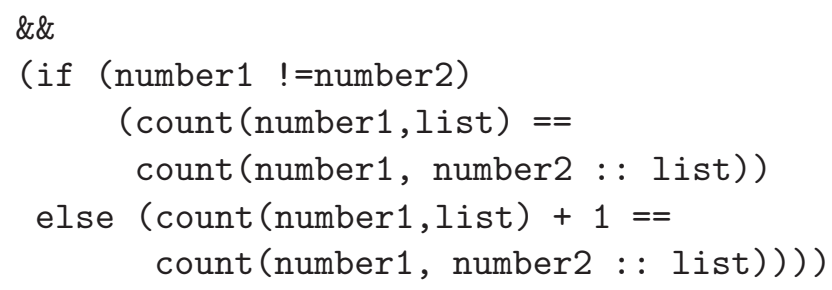

An interesting feature of this property is that it is a complete specification of our counting function. In fact, it is surprisingly close in what it says to what the actual code of our implementation says in its text.

Exercise 5.2 Explain the justification for equivalence of the following sequence of the properties to the last property stated above ("Count specification (A)"):

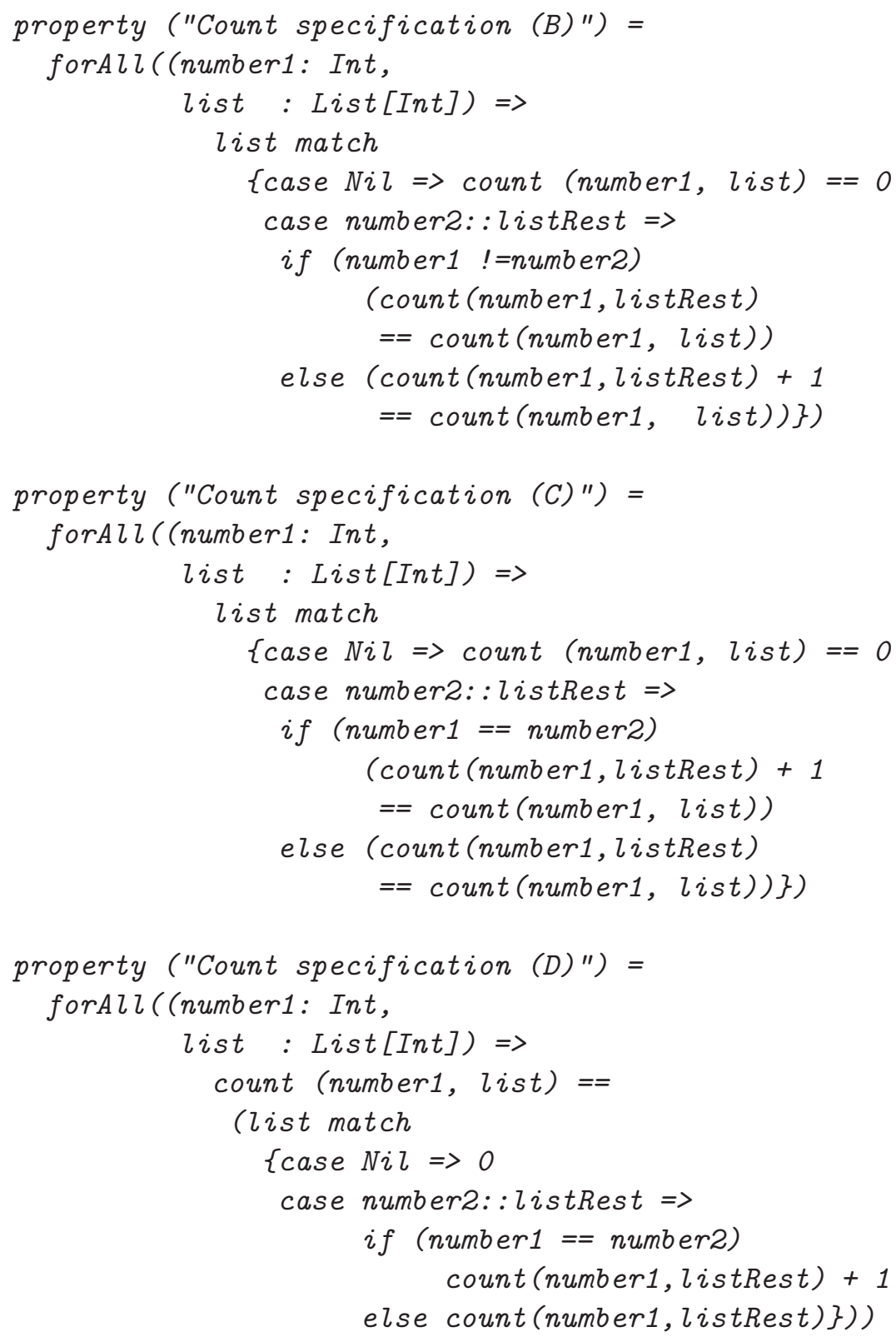


It is both a good thing and a bad thing when the most intuitive properties that we can think of for our functions are essentially the same as the program that we use to implement it. It's a good thing because it increases the chances that our implementation has these properties. It can be a bad thing for a couple of reasons. First, it could mean that we have not identified enough other properties of our function to allow us to double-check the core set of properties that we are using for defining this property. Second, it can mean that we may have made the same mistake twice in both specifying and implementing our function. The solution to the first problem is to continue thinking about properties that we would like our function to have. An example of such a property is the "Count/append" property that came across at the start of this example. The solution to the second problem can be to arrange for another team member who has not seen the code or solution to independently come up with the specification of the properties that they would like this function to have.

\section{Functions with an inverse}

While we have barely scratched the surface of specifying properties of single functions, it is important to move on and to consider what happens when we are developing multiple functions that have interrelated properties. There are numerous patterns of such interactions. In this section, we will consider a simple example of a very common pattern, namely that of functions that have an inverse. One of the nice features of this pattern is that it is easy to intuitively identify in many areas of computer science. Intuitively, anything that we can view as a kind of "encoding" is an example of such a property because we generally do not use that word unless there is both an encoding and a decoding function, and where the second function serves as an inverse to the first.

\subsection{Encoding integers as bits}

As a simple example of this kind of pattern, we will consider the problem of converting from nonnegative integers (natural numbers) into binary and back. This problem can be informally specified as follows: if we are converting decimal numerals then we would be turning a value like 125 into List $(5,2,1)$. We actually reverse the order and have the least significant digit come first in the list because that makes the functions just a bit more convenient to write. In any case, what we want here is a binary encoding. So, for an integer like 6 that is represented in binary by 110 we will want to get back the List $(0,1,1)$. In fact, because with binary digits we only have two choices of digit, we want the result to use bools and represent 0 with false and 1 with true, so that the result for the example above is List(false, true, true).

\subsection{Use cases for encoding}

As usual, we start with writing down examples, and we can write the function immediately after writing the examples. In both of the following cases, ScalaCheck tells us that both functions have passed our tests with flying colors:

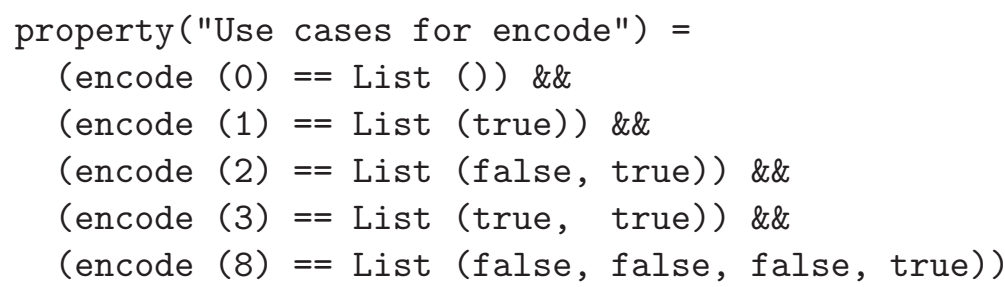




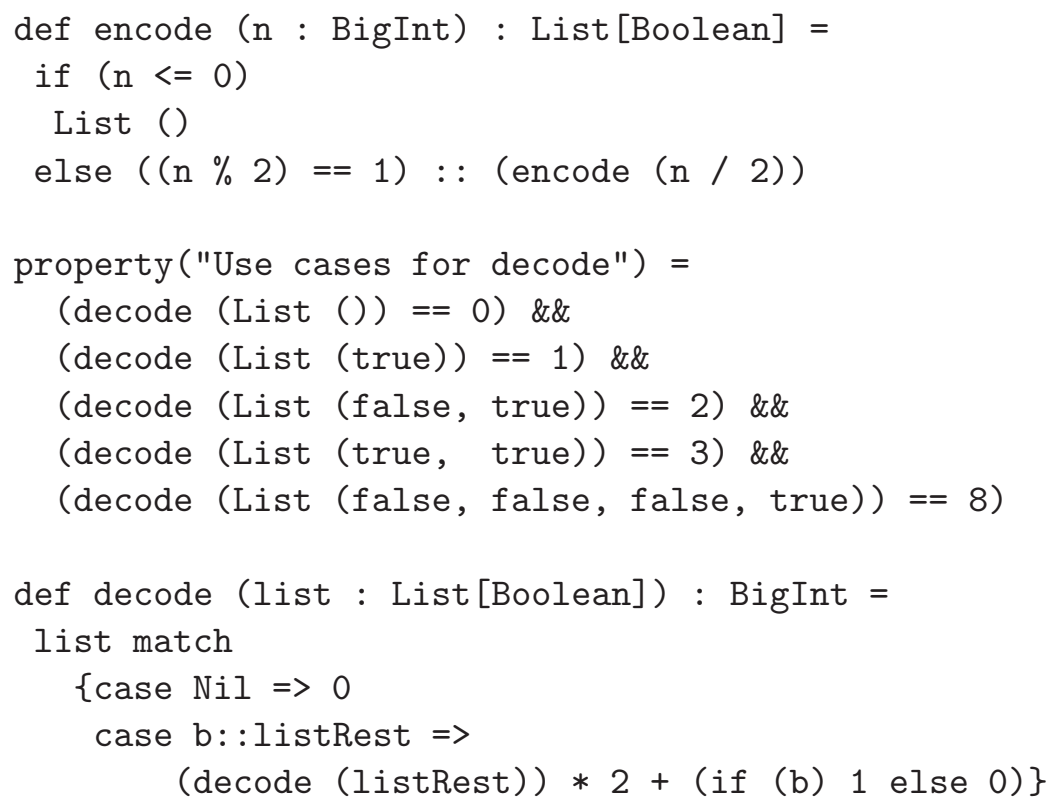

In the case of the two functions above, the encoding and decoding functions seem so simple that it is hard to imagine that anything can be wrong with them. However, even if the functions appear to provide precisely the functionality that we need, it is useful to explore the properties of these functions to make sure that we also understand the functionality that we have just provided. As a first example, we will state the property that encoding followed by decoding produces the same value we started with. That is,

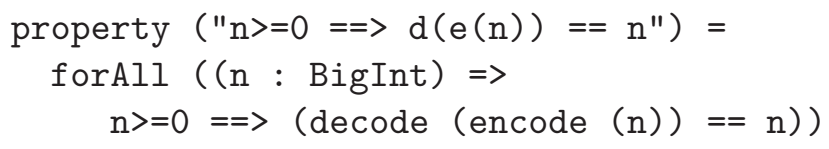

While this property holds for this function, it is useful to note that the dual property does not hold. That is, decoding following by encoding does not produce the same binary digit. This may seem a bit curious, but in fact this is a common situation for many encoding/decoding pairs.

\subsection{The asymmetry of invertible functions}

Expressing this property in ScalaCheck allows us to find a counter example:

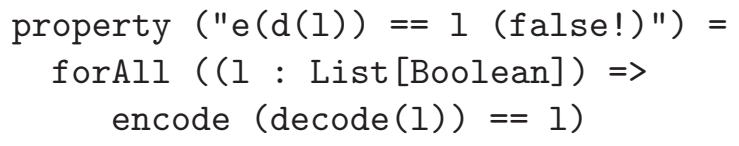

Testing this property quickly returns a counter example that gives us a good idea of why it does not hold:

! SmallExamples.e $(\mathrm{d}(\mathrm{l}))==1$ (false!): Falsified after 6 passed tests.

$>$ ARG_0: List("false")

What has happened is that in our encoding the number 0 is represented by the empty list. Decoding works fine on the empty list to produce 0 . Moreover, it will also produce that same value for any list that 
is all zeros. But for all such lists, the encoding function will always be only the empty list, which is not really the same list as all the lists of all zeros. In fact, in general, applying our decoding and encoding functions will always have the effect of removing all leading zeros. And so they don't always give us back exactly what we started with, which is what the property above says.

It is useful to note that decoding followed by encoding does give us back what we started with if what we started with was produced by the encoding function. This can be expressed as follows:

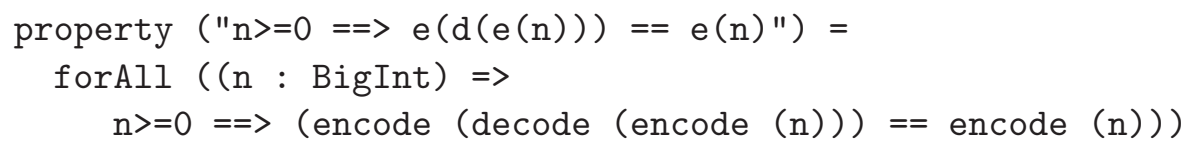

Thus, the key property of such encoding systems is that the encoding function is an inverse of the decoding function, which is what is captured by the first property. This is a pattern that we will see in other computing applications that go by names such as encryption, serialization, parsing, and others.

Exercise 6.1 Explain why it may not be particularly surprising if the property:

$$
" n>=0==>d(e(n))==n "
$$

then the property

$$
" n>=0==>e(d(e(n)))==e(n) "
$$

also holds.

Exercise 6.2 (Lab) While it is the case that an encoding function is not always an inverse to the decoding function, it is worthwhile to note that this can be a useful property when it holds. In particular, it means that every target representation is a unique encoding of a number in our source for the encoding.

Write the code and properties for an encoding/decoding pair from integers to lists of booleans and where this symmetry does hold. Hint: Treat the empty list as always holding an implicit true at the most significant digit, and subtract one from the interpretation so that 0 is still representable.

Exercise 6.3 (Lab) Define an addition function on the original encoding of integers. State its correctness property, and check it using ScalaCheck.

\section{Acknowledgments}

We would like to thank the participants of the summer school that was organized at Halmstad University from May 30th to June 1st. A special thanks also goes to the fourth speaker the summer school, Prof. John Hughes. The goals of the workshop would not have been achieved without the active efforts of the faculty of Halmstad University that worked closely with the authors to incorporate these ideas into the undergraduate and graduate curricula, especially Nicolina Månsson, Elisabeth Uhlemann, Ulf Holmberg, Tony Larsson, and Thorsteinn (Denni) Rögnvaldsson. Prof. Page's visit to Halmstad was greatly facilitated by the hard work of Eva Nestius, Magnus Jonnson, Bertil Svensson, and Magnus Larsson. Finally, Paul Brauner kindly provided us with helpful comments on a draft of these notes.

Rex's visit would have not been possible without the generous support of the U.S. Department of State through the Fullbright Scholar program, as well as the support of Halmstad University. 


\section{References}

[1] Accuracy and Precision. Wikipedia, The Free Encyclopedia. Available online from wikipedia.org. Viewed May 2011.

[2] Gojko Adzic (2011): Specification by Example: How Successful Teams Deliver the Right Software. Manning, Greenwich, CT.

[3] Robert Cartwright (1981): Formal Program Testing. In: Principles of Programming Languages, pp. 125-132, doi $10.1145 / 567532.567546$.

[4] Koen Claessen \& John Hughes (2000): QuickCheck: A Lightweight Tool for Random Testing of Haskell Programs. In: International Conference on Functional Programming, pp. 268-279, doi:10.1145/351240.351266.

[5] ScalaCheck Tutorial. Available online from http://code.google.com/p/scalacheck/wiki/UserGuide. Viewed May 2011.

[6] Use-case Analysis. Wikipedia, The Free Encyclopedia. Available online from wikipedia.org. Viewed May 2011.

\section{A Broader Educational Context}

The seed for this tutorial was planted when Rex organized a workshop on Teaching Software Correctness in May 2008. Walid attended the workshop and was struck by the unusual effectiveness of propertybased testing in helping programmers develop a program and a property that can actually be verified mathematically by a theorem prover. While it was impressive to see the theorem prover used in the workshop (ACL2) to automatically prove a host of sophisticated properties, what was most impressive was the effect that the combination of property-based testing had on the programmer: property-based testing guided the programmer to a correct program, which is really the only plausible candidate for even starting to think about the completely independent, non-trivial, and often quite labor-intensive task of proving a program correct.

As luck would have it, two years later, in May 2010, Rex was organizing the Symposium on Trends in Functional Programming, which Walid attended. At that meeting, Walid mentioned to Rex that he was moving to Halmstad University, which is in the process of starting up an independent Ph.D. program, and many of the faculty are genuinely interested in curricular development. Thanks to a Fulbright Scholarship, exactly one year later, Halmstad University hosted Rex for a one-month visit aimed at introducing his ideas into the Embedded and Intelligent Systems curricula at both the graduate and undergraduate levels.

Working on exploring how this can be achieved started by a kickoff meeting that was organized the first week of the visit, which was well attended by faculty. It included a presentation of the bachelor's program (three years) by Nicolina Månsson and of the masters program (two years) by Jörgen Carlsson, followed by a presentation by Rex of the key ideas in his approach. Both in terms of research and education, the strengths of Halmstad University's programs considered to be:

- Multicore architectures

- Real-time communications

- Cooperative embedded systems

- DSLs and program generation

- Modeling and simulation of cyberphysical systems 
The effectiveness of both research and education of these areas would be strengthened if students were more capable of producing high-quality software. It was therefore instructive to learn of the results of the careful analysis that Rex had conducted of a wide range of undergraduate curricula in the U.S., as well as his varied efforts to introduce more "property-based thinking" into various courses. For example, it seemed that students tend to find that introducing testing-based methods into math classes makes them easier. On the other hand, introducing these ideas into software engineering gets students' attention primarily because of its novelty. Potential employers of students seem to easily appreciate the value of getting students who are trained to rigorously test their programs, and who are capable of producing high-quality software. It was also interesting to learn from Rex that there are studies that show that testing techniques based on analysis of the code itself are generally much more economically viable than ones based solely on behavior.

Two general problems with computer science curricula were identified. The first is that the theory classes, such as discrete math classes, tend to be disconnected from the artifacts that students in this discipline are most interested in, such as software and hardware. The other problem is that the courses that teach important theoretical tools, such as logic for example, tend to focus on the meta-theory of such tools rather than on how to use them in relation to concrete artifacts that students are interested in (again, software and hardware). In what class do we learn how to use logical statements to state mathematical properties of programs, not to mention learn to reason about programs in terms of such properties?

It was suggested that the most practical and possibly most effective approach will be to incorporate Rex's ideas into the curriculum by injection into a variety of different courses. Also, it was suggested that using property-based testing could be an effective vehicle for introducing a wide range of concepts relating to software correctness and logic. With such a foundation, students are prepared to read books or follow courses that are more mathematically oriented, either on their own or in the context of advanced courses offered at the university.

Several candidate areas for introducing these ideas where identified. In the master's degree program, where books and instruction are in English:

- Embedded systems programming course (using ScalaCheck and maybe ACL2)

- Cooperating intelligent systems course

- Discrete math course

- Cyber-physical systems (CPS)

Several specific ideas were discussed for CPS. Natural candidates included scheduling problems. Some specific ideas: 1. number of steps in a computation (e.g. interrupt handler), 2. model interrupt handler as an ACL2 computation + step count, 3. prove bound on the number of steps, 4. example: moving window algorithms, 5. bounded work to update, and 6. d.s. maintains an invariant. All that would be needed would be two examples of this, and to create material for a lecture + homework. This can be packaged as a one-week module, which may also be usable in other courses.

In the bachelor's program, where books and notes are in Englsih but instruction is in Swedish:

- Programming I, teaching specifications together with programs.

- Programming II, teaching contracts

- Switching theory and digital control.

- Algorithms and data structures

- Computer systems organization 
This suggested that there should be no shortage of opportunities for introducing Rex's ideas at Halmstad. With this, it was agreed that Veronica, Rex, and Walid would focus on preparing materials for the workshop that would serve as practical starting points for the faculty at Halmstad to explore these possibilities more concretely. These are the notes presented in this document.

The rest of the story will depend on how well we succeed at integrating these ideas into various courses in the Halmstad curriculum! 